\title{
Star Formation in the Molecular Cloud Associated with the Monkey Head Nebula: Sequential or Spontaneous?
}

\author{
J. O. Chibueze ${ }^{1}$, K. Imura ${ }^{1}$, T. Omodaka ${ }^{1}$, T. Handa ${ }^{1}$, T. Nagayama ${ }^{2}$, \\ K. Fujisawa ${ }^{3}$, K. Sunada ${ }^{2}$, M. Nakano ${ }^{4}$, T. Kamezaki ${ }^{1}$, and \\ Y. Yamaguchi ${ }^{1}$ \\ ${ }^{1}$ Department of Physics and Astronomy, Graduate School of Science and Engineering, \\ Kagoshima University, 1-21-35 Korimoto, Kagoshima 890-0065, Japan \\ email: james@milkyway.sci.kagoshima-u.ac.jp \\ ${ }^{2}$ Mizusawa VLBI Observatory, National Astronomical Observatory of Japan, 2-21-1 Osawa, \\ Mitaka, Tokyo 181-8588, Japan \\ ${ }^{3}$ Department of Physics and Informatics, Faculty of Science, Yamaguchi University, Yoshida \\ 1677-1, Yamaguchi 753-8512, Japan \\ ${ }^{4}$ Faculty of Education and Welfare Science, Oita University, Oita 870-1192, Japan
}

\begin{abstract}
We mapped the $\mathrm{NH}_{3}(1,1),(2,2)$, and $(3,3)$ lines of the molecular cloud associated with the Monkey Head Nebula (MHN) with 1'.6 angular resolution using Kashima $34 \mathrm{~m}$ telescope. Its kinetic temperature distribution was contrary to what is expected for a molecular cloud at the edge of an expanding H II region and suggested that the massive star associated with S252A compact HII region formed spontaneously rather than through a sequential process.
\end{abstract}

Keywords. HII regions — ISM: clouds — ISM: molecules

\section{Introduction}

Massive star formation is yet a poorly understood concept in astronomy. Elmegreen \& Lada (1977) proposed that massive stars in OB association form sequentially, triggered by the shock effect of an expanding H II region. To observationally verify the accuracy of this scenario, we studied the Gem OB1 cloud, a dense molecular cloud at the edge of an diffuse H II region (Monkey Head Nebula, MHN).

\section{Observations and Results}

We mapped the $\mathrm{NH}_{3}(1,1),(2,2)$ and $(3,3)$ lines of the dense molecular gas of Gem OB1 from which we estimated its mass and kinetic temperature distribution. We obtained both the virial and the LTE mass to be $\sim 2000$ solar masses. We expected higher temperatures at the interface between the hot ionized gas of the H II region and the molecular cloud, but on the contrary, temperature range $10-30 \mathrm{~K}$ was dominant in the entire region and $>50 \mathrm{~K}$ in the region near S252A. 8.4 GHz Yamaguchi $32 \mathrm{~m}$ telescope observation of the ionized gas showed the true morphology to be similar to that of its optical image. Thus, the influence of the expanding $\mathrm{H}$ II region, MHN, is quite limited and the warmer gas is heated up by the S252A compact H II region. Comparing this with the infrared data, we concluded that the massive star of S252A formed spontaneously, not sequentially.

\section{Reference}

Elmegreem, B. G. \& Lada, C. J. 1977, ApJ, 214, 725 